\title{
Pterional Approach
}

\author{
Sophie Peeters and Julius July
}

\section{$1.1 \quad$ Introduction}

For years, surgeons have been developing surgical approaches attempting to achieve maximal surgical exposure with minimal brain retraction, advantages found within the frontotemporal or pterional approach, first described by Yasargil, four decades ago [1-3]. Compared to its predecessors, this approach allowed for wider frontobasal exposure, secondary to more significant drilling away sphenoid wing; in addition to that surgeon could dissect and split Sylvian fissure wider [1]. Consequently, it was applicable for clipping of both basilar tip and anterior circulation aneurysms, with an excellent safety-efficacy profile [1]. Later on, the approach will be modified and combined with others, and its indications are only growing in number.

\section{S. Peeters}

Department of Neurosurgery,

Faculty of Medicine Universitas Pelita Harapan (UPH), Neuroscience Centre Siloam Hospital Lippo

Village, Tangerang, Indonesia

Department of Neurosurgery, University of California Los Angeles (UCLA), Los Angeles, CA, USA

J. July $(\bowtie)$

Department of Neurosurgery, Faculty of Medicine Universitas Pelita Harapan (UPH), Neuroscience Centre Siloam Hospital Lippo Village,

Tangerang, Indonesia

\subsection{Steps of the Approach}

\subsubsection{Positioning and Preparation}

Position the patient supine, head fixated with a Sugita or Mayfield head holder. Two pins need to be contralateral at superior temporal line right above the temporal muscle, and lastly, the third pin is placed at the ipsilateral mastoid (Fig. 1.1).

When positioning the head, pay attention to five movements: (1) traction when moving the head toward the surgeon; (2) lifting the surgical area to a level above the atrium, to avoid impeding venous return; (3) deflection and (4)

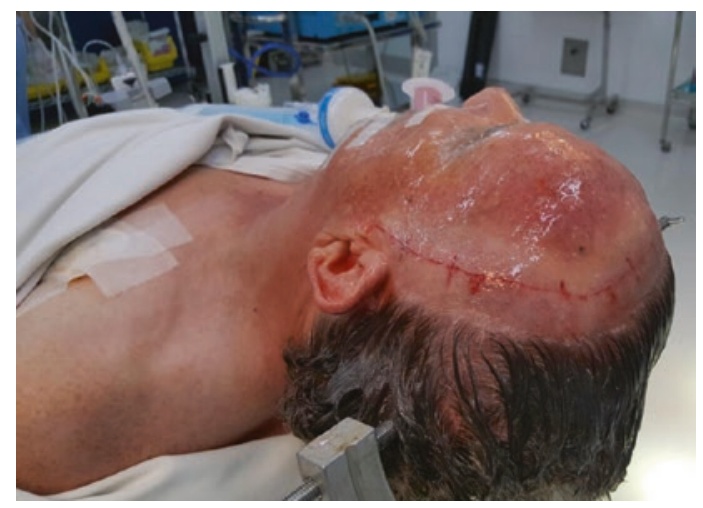

Fig. 1.1 Supine position, head is fixed with Sugita head holder with three pins fixed. The angle of head rotation can be tailored according to tip of exposure that we want to achieve. Usual angle for pterional approach would be around $30-60^{\circ}$ head rotation to the contralateral side 
rotation, both related to the surgical indication; and (5) torsion, with the angle between the head, neck, and shoulder, large enough to give the surgeon a satisfying lateral operating position [4]. Conditions needing slight deflection with rotation $45-60^{\circ}$ from vertical line to the floor include cavernous sinus pathologies and basal lesions, for example, internal carotid (IC)-ophthalmic aneurysm, IC-posterior communicating aneurysm, and IC-choroidal segment aneurysm. Contrarily, conditions requiring increased deflection with rotation $30-45^{\circ}$ from vertical line to the floor include carotid bifurcation aneurysm, middle cerebral artery (MCA) aneurysm, anterior communicating aneurysms, and anterior cerebral artery aneurysm, as well as suprasellar tumors.

The hair is combed with chlorhexidine solution $\left(\right.$ Microshield $^{\circledR}$ ) or any detergent solution and subsequently shaved about $2 \mathrm{~cm}$ from incision line. The shaved skin is then treated with alcohol, getting rid of scalp fat and facilitating sticking of the sterile fields. The incision is marked with methylene blue or marked with transofix (Fig. 1.1) after injection of local anesthetic with lidocaine $10 \mathrm{mg} / \mathrm{mL}$ mixed with adrenaline $6.25 \mu \mathrm{g}$ solution. The injection at pin site and the incision site provides additional protection to pain, and the adrenaline would help to keep the drape clean from the blood loss during incision.

An arcuate semicoronal frontotemporal skin cut is performed starting from the upper edge zygomatic arch, anterior to tragal cartilage. The skin cut should preserve the superficial temporal artery and frontal branch of facial nerve. The incision extends to the midline of the frontal skull, ideally concealed behind the hairline. The arch of Sugita head holder should be placed for suspension and traction of myocutaneous flaps to avoid compression of the eyeball. Finally, incise the skin with knife, and stop the scalp bleeding with bipolar, and apply the Raney clips. Proceed with subgaleal dissection until the line between the superior border of orbital rim and tragus dissects until leaving anterior one fourth of the temporal fascia [5]. Retract the skin flap to anterior, and hold it with fish hooks.

\subsubsection{Dissection and Mobilize Temporal Muscle}

Interfascial splitting at temporal region was first introduce by Yasargil [6]. The challenge of this step is preserving the frontalis nerve and reducing unwanted cosmetic results after the surgery. The temporal muscle can be divided into two portions: one originating along the superior temporal line and then inserting to mandibular coronoid process and the second portion originating on the temporal squama and inserting onto the mandibular temporal crest [7]. There are also two layers of fascia that cover this muscle (superficial and deep). Deep layer protects the vessel supplying the muscle which consists of anterior, intermediate, and posterior deep temporal arteries, all originating from the maxillary artery. It also protects the innervations that are coming from trigeminal nerve (temporal and mandible branches) [7]. The splitting plane through laminae of the temporal fascia is created, protecting the frontotemporal branch of the frontal nerve [6]. The temporal muscle is retracted away from temporal fossa, thus improving exposure and limiting unnecessary retraction of the cortex while doing surgery. Dissect the superficial fascia vertically with a scalpel of Metzenbaum scissors starting about $2 \mathrm{~cm}$ at superior orbital rim to the zygomatic root, along superior temporal line [7]. Avoid monopolar cauterization to limit the risk of temporal muscle atrophy. Placing a hook in the center may aid the separation by scalpel of the superficial fascia with the underlying fat plane, obtaining best identification of the deeper muscle part that contains nerves and vessels. Both the superficial and fat layers are reflected anterolaterally over the skin flap. To detach the temporalis muscle, first use the monopolar (on coagulation to avoid any extra bleeding), and transversally cut the temporal muscle at its insertion, medially to zygoma and frontal zygomatic process. Then, use a scalpel to cut the muscle in an anterior to posterior motion slightly below the superior temporal line. A small portion of the myofascial cuff is intentionally left at superior temporal line on the bone flap for future muscle reconstruction in order to prevent atrophy of the muscle $[8,9]$. 
Secondarily, detach the deep fascia from the skull using a Cushing's elevator. To avoid bleeding, start detaching the muscle flap inferiorly where the muscle is least attached to the bone [9]. These maneuvers release the fascia temporalis, aponeurosis, temporal muscle, and temporal periosteum from the temporal muscle's origin. Once detached, the temporal muscle is retracted inferiorly leaving its tendinous attachment intact, exposing the pterional region. Finally, reflect the periosteum cranial to superior temporal line to the anterior direction. Remember to keep all the tissue moisture by covering it with wet gauge.

\subsubsection{Craniotomy}

The craniotomy for this will vary based on surgical aim; typically, one or two burr holes are sufficient, though many surgeons will use three. The aim of this craniotomy is more basal exposure as well as generous visibility of the Sylvian fissure. Thus, the goal is to attempt exposure of the inferior frontal, part of the middle frontal, gyrus superior temporal, and superior part of gyrus middle temporal. The burr holes are performed with common drilling system aiming posteriorly and inferiorly to avoid penetrating the orbit [9]. The first and most important burr hole, also called "keyhole," is placed at the meeting point between zygomatic bone, the superior temporal line, and supraorbital edge [9]. Second trepanation is located inferiorly in the temporal bone's squamous portion, near the pterion. Lastly, the third hole, optional but recommended in older patients due to their dura sticking to the skull, is made on the most posterior end of superior temporal line. Use dissectors to detach the dura from the skull. The Sylvian fissure lies beneath the pterion; thus, the inferior frontal gyrus lies between the pterion and the superior temporal line. Use either a Gigli saw or a craniotome for the craniotomy, as long as the cut is made along the outer edge of the trepanations and extended frontally, taking care not to open the frontal sinus. Use a bipolar forceps for hemostasis at low power to prevent more dural shrinking. Greater sphenoid wing lies deep between first and second burr holes and, if too prominent, may require some rongeuring prior to elevate the bone flap; the alternative is fracturing the bone when elevating the flap.

\subsubsection{Basal Drilling}

Extradural gentle drilling of greater sphenoid wing process, projections of orbital roof, and the rest of temporal squama until exposing the superior orbital fissure may be necessary for better access to the basal structures with limited brain retraction and flatter bone ridges [8]. The exposure will be magnified even more with opening of the cisterns and subsequent drainage of the cerebrospinal fluid. Some even recommend performing lumbar spinal CSF drainage and ventriculostomy to get more relax brain for a skull base approach. Use dissectors to detach the dura from orbital roof and part that left on lesser sphenoid wing bone without extending past the limits of the drilling, to avoid forming extradural dead spaces. Place a spatula, attached to an orthostatic retractor, to serve a shield to protect the dural surface. A cylindrical or round drill is used to remove remaining bony prominences from the outside inward on the orbital roof and the remaining temporal squama. Next, protect the underlying dura with a spatula, and drill the lesser sphenoid until dural cuff containing the meningoorbital artery located at the superolateral border of the superior orbital fissure is visible. Isolate, coagulate, and section this artery using a scalpel. The best drill for delicate drilling is diamond drill. To relax tension on the dura, if necessary, a small incision at the projection of the lateral fissure level can allow for cerebrospinal fluid drainage. After drilling, anchor the dura by stitching it (3.0 round silk) at bone edge to avoid collection of epidural blood during or after the operation.

\subsubsection{Opening the Dura and Exposing the Brain}

The purpose of incising and retracting the dura is to keep it smooth when folded back over the bony surface; dural folds can sometimes hinder visibility. 


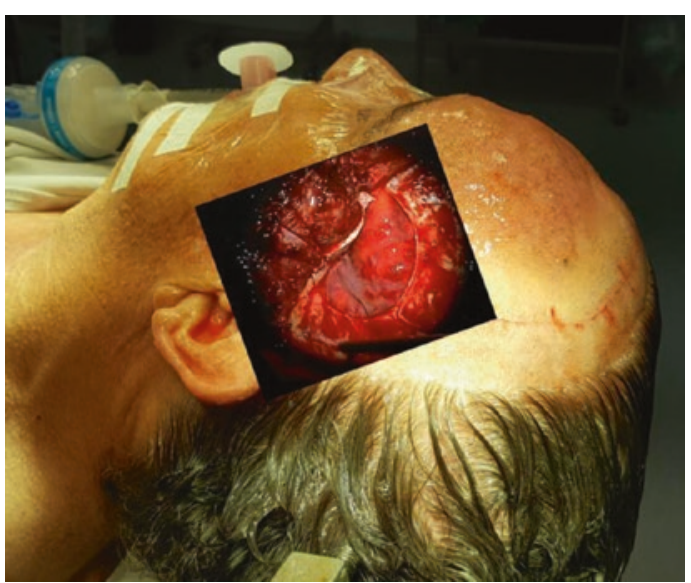

Fig. 1.2 The inserted picture shows the opening flap is retracted toward anterior inferior using fish hook and the C-shape opening of dura with its concave facing the skull base. The brain is slightly tense and red, which is common for aneurysmal subarachnoid bleed. This is the case with left M1 bifurcation aneurysm bleed

Use Metzenbaum scissors to incise the dura in a "C"'-shaped manner creating a flap pedunculated toward the previously roungered greater sphenoidal wing, improving parasellar visibility.

The concavity of the incision should be toward the orbit and sphenoid that were previously roungered or flattened using high-speed drill; the pedunculated flap is then retracted over the greater sphenoidal wing remains (Fig. 1.2). This maneuver will exposes the inferior frontal gyrus, the superior temporal gyrus, the middle temporal gyrus, and most importantly the Sylvian fissure.

\subsubsection{Splitting Sylvian Fissure}

Sylvian fissure can be divided into superficial and a deep part [4]. The stem of the superficial part extends medial to the semilunar gyrus of the uncus between inferior frontal lobe and pole of temporal lobe until lateral portion of sphenoid wing. The stem then split into anterior ascending branches, anterior horizontal branches, and posterior branches. The deeper part of the lateral fissure divides into an anterior part (sphenoidal) and posterior part (operculoinsular). Anterior part is the narrow space between frontal and temporal, located behind sphenoid ridge; it commu- nicates directly cistern around the carotid. The posterior part is made up of two clefts-opercula and insular. Insula becomes visible once the Sylvian fissure's lateral walls are separated. It links the temporal lobe with posterior orbital gyrus through limen insula, a threshold between the fissure laterally and the carotid cistern medially. The insula covers up a set of valuable structures such as internal capsules, external capsule, extreme capsule, the claustrum, the basal ganglia, and the thalamus. Typically, the basal cisterns are opened, prior to the transsylvian dissection, in order to relax the brain through cerebrospinal fluid drainage. This allows for a cisternal approach to aneurysms, first presented by Yasargil [1]. The opening of the Sylvian fissure is started near the pars triangularis of the parasylvian inferior frontal gyrus, where there is the most room between frontal and temporal lobes [4, 9] (Fig. 1.3).

Using microscissors and either a scalpel blade number $11,25 \mathrm{G}$ needle, or diamond knife, the superficial portion of Sylvian fissure is cut and dissected starting from the frontal part of superficial Sylvian vein. The latter typically runs laterally toward the distal end of the temporal lobe and then drains into the sphenoparietal sinus. However, in some cases, the superficial Sylvian vein may drain toward the frontal Sylvian vein and finally into the superior sagittal sinus, justifying a dissection lateral of the superficial Sylvian vein. Relevant anatomical structures identifiable at this stage are ICA, MCA, ACA, and the optic nerve. It is very important to have a good dissec-

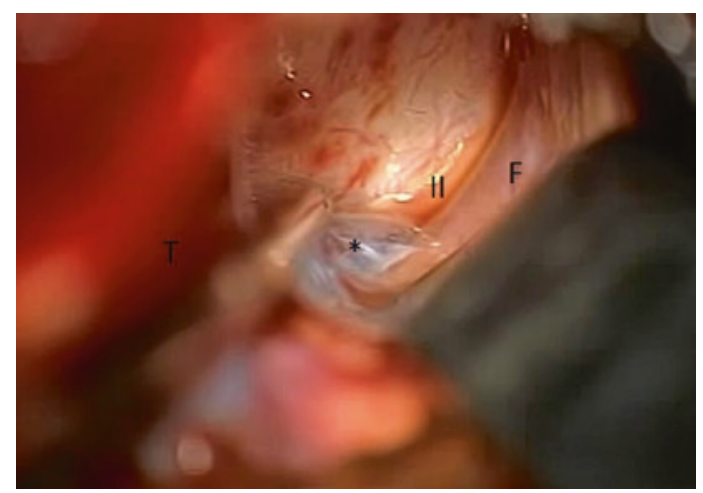

Fig. 1.3 The pars triangularis of the parasylvian inferior frontal gyrus (*), $F$ frontal, $T$ temporal, $I I$ optic nerve 

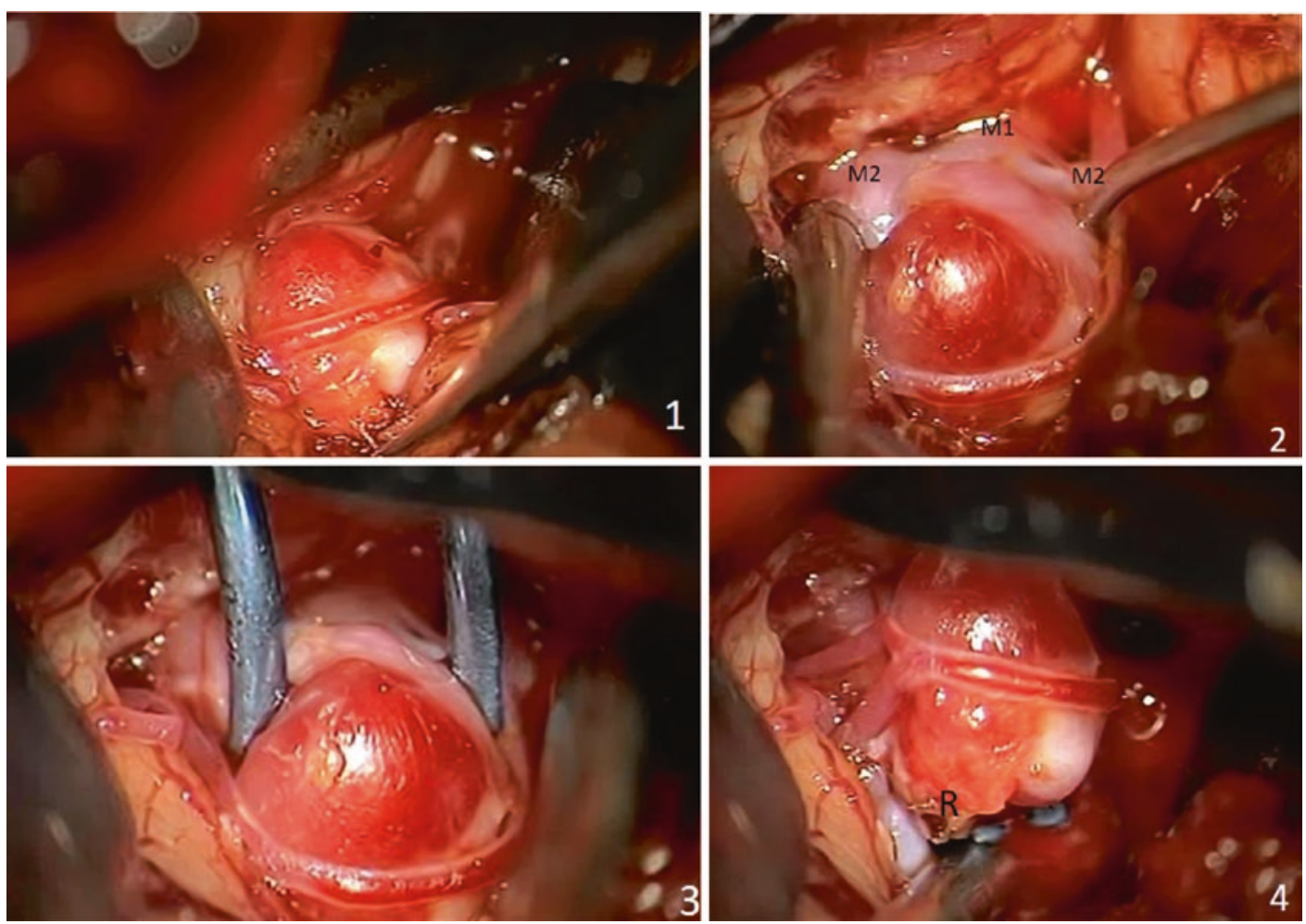

Fig. 1.4 The case of M1 bifurcation aneurysm. (1) The aneurysm was identified, but it's not well exposed yet, and it's necessary to continue dissection to identify all the surrounding, especially to find the parent artery in case the surgeon needs temporary occlusion. (2) Sylvian fissure dissection allows surgeon to expose the M1, M2, and branches. (3) Avoiding the branches and putting the clip on the aneurysm neck. (4) Expose the legs of the clip, and confirm the aneurysm is well clipped ( $R$ rupture site)

tery and sponge tamponade methods. After closing the dura until watertight, the bone flap is repositioned and secured with miniplates. The burr hole area could be covered with a titanium clamp, or the other options are to fill the hole with bone dust and make sure that it's covered well with periosteum. The periosteum is very important for bone healing and remodeling to the normal appearance. The temporal muscle flap is placed where it belongs, and the temporal muscle is re-sutured to the remaining myofascial cuff on the skull, while the reflected fascia is sutured to the fascia-periosteum complex. Finally, the fascial incision and the scalp incision are closed in the usual manner. The choice of an extradural subgaleal closed suction drain should enhance the healing and reduce fluid collections under the flap [10].

The classical pterional approach is attractive and widely used for many reasons, including smaller size craniotomy yet still allowing a wide 

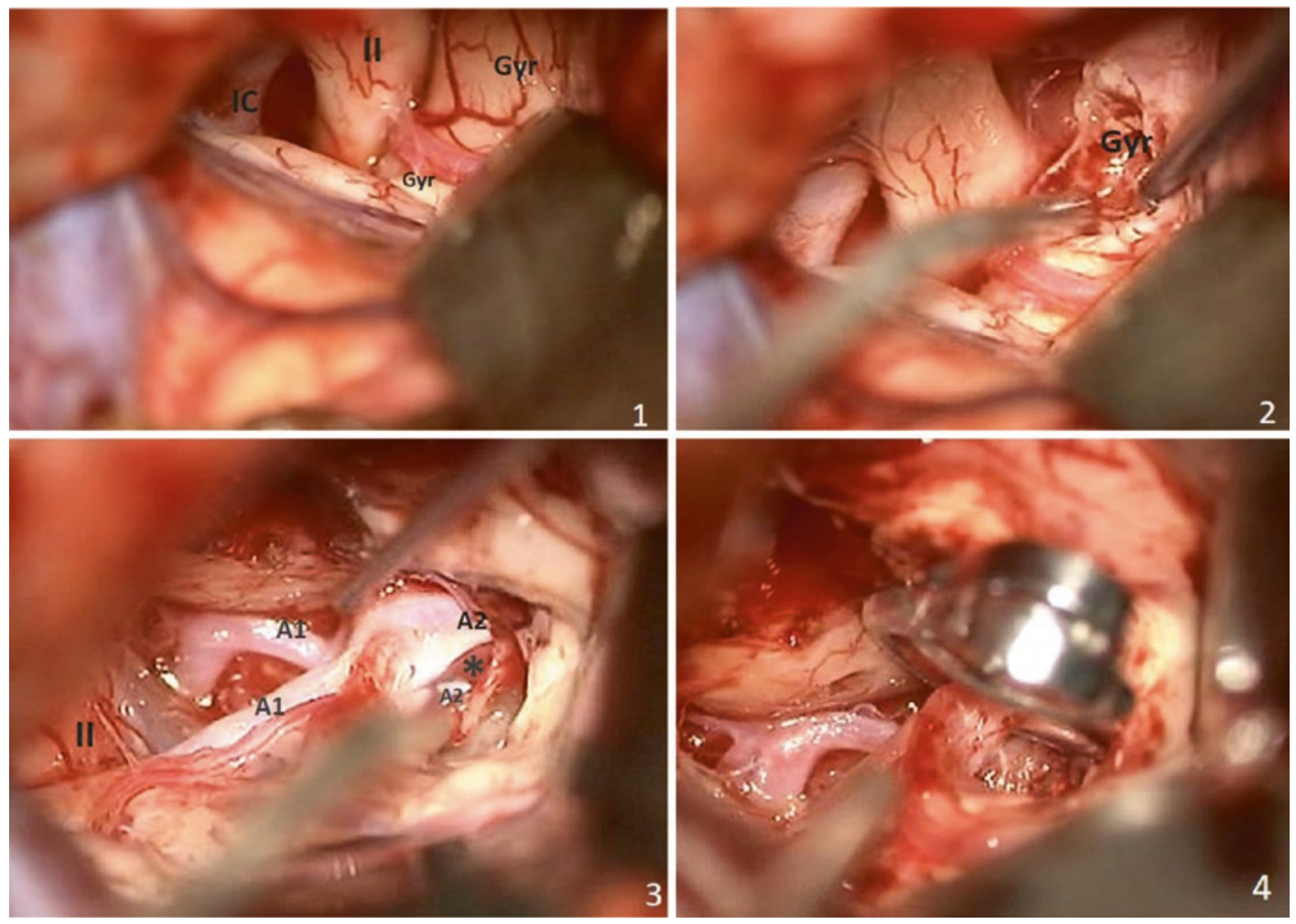

Fig. 1.5 The case of Acom aneurysm rupture, with left pterional approach. (1) The gyrus rectus obscures the AcomA complex. (2) Partial resection of gyrus rectus

frontobasal exposure and rapid access to basal cisterns and the circle of Willis, without requiring extensive brain retraction. The approach limits unnecessary frontal and temporal lobes exposure. It also prevents risk of injury to the optic radiations or uncinate fasciculus, thus avoiding postoperative visual field deficits and aphasia, in the context of temporal lesion removal [11]. On a cosmetic level, the pterional approach was favorable for its good skin incision behind the hairline, appropriate size bone flap, and the osteoplastic craniotomy preventing some of the postoperative temporalis atrophy.

\subsection{Indications of the Approach}

Initially, the approach was suggested for most of microsurgical approach for cerebral aneurysms in the whole circle of Willis, particularly in the anterior circulation. Later, the pterional approach
(Gyr). (3) Exposing the AcomA complex with the aneurysm between the both A2. (4) Clipping with ring clip to spare the $\mathrm{A} 2$ on the ipsilateral side

was also used for the removal of tumors and other vascular malformations found in cavernous sinus area but also sella, parasellar, temporal, subfrontal, and anterior and anterolateral midbrain regions. Examples of lesions successfully treated through this approach include aneurysms, olfactory groove meningiomas, cavernomas of the anterior mesiotemporal region, temporal horn tumors, and a clival chordoma. It's still one of the most commonly used approaches in neurosurgery practice.

\subsection{Limitations of the Approach}

The main factors to be considered when selecting the right extent of exposure required are compartment that need to be exposed and of course the location and size of aneurysm. The main issues regarding the pterional approach are obstruc- 
tion of the operator's visualization by the basal structures, such as orbital roof, sphenoidal ridge, frontal and temporal lobes, and ICA, and temporal muscle. Sometimes surgeon need to work at a deep and narrow naturally available space between the carotid artery and optic or oculomotor nerves [1]. Hence, though the indications for a pterional approach are numerous and it is a widely applicable technique, it is not without limitations, typically exposure-related. The pterional approach is now often modified by or combined with the following approaches, temporopolar, pretemporal, cranioorbitozygomatic (middle cranial fossa lesions or upper clivus, upper basilar complex), and lateral supraorbital, in order to obtain the optimal diameter and angle of exposure for the pathology being treated. For example, if clipping an aneurysm of the posterior circulation (i.e., basilar tip aneurysm), the surgery may have to include removal of the anterior plus or minus the posterior clinoid processes for better visibility; or in order to have better access to the parasellar region, reshaping of the zygomatic complex may be helpful [7]. Additionally, ipsilateral vessels and the gyrus rectus can sometimes hinder ideal exposure of the AcomA complex and the contralateral vessels in a pterional approach; the orbitopterional approach is a great alternative. If a more inferior temporal view is required, removal of the zygomatic arch may be considered.

\subsection{Complications and How to Avoid}

The main complications that can be expected from this approach are aneurysmal intraoperative rupture, epidural or subdural hematoma, infection, ischemic events, early or late rebleeding, temporal muscle atrophy and/or dysfunction, and lastly, cosmetic cranial defects.

The interfascial flap dieresis technique from the original pterional approach, as described by Yasargil, was found to be associated with more prevalent, worse, and longer-lasting palsy of frontotemporal facial nerve branches, temporalis muscle atrophy, and temporomandibularis joint dysfunction [12]. The aim of this two-layer flap is maximizing visibility at the expense of severe retraction of the temporalis muscle. On the other hand, the myocutaneous flap has considerably less risk for facial nerve injury since temporal muscle is lifted up with scalp in a one-layer flap $[5,13]$. However, this myocutaneous flap is at the detriment of worse exposure secondary to the bulkiness of the temporalis muscle [6]. Kim and Delashaw propose an osteomyoplastic monobloc technique in order to prevent both cosmetic and functional undesirable outcomes [14]. The procedure spares the temporalis muscle and the basal bone. It involves dissecting a subperiosteal detachment below the temporal separating it from the underlying bone while maintaining the muscle's attachment to superior temporal line and temporal squama. This is followed by the creation of a keyhole $2 \mathrm{~cm}$ posterior to pterion at the Sylvian fissure line. Final step consists of roungering part of the sphenoid wing allowing its removal with the pterion in one piece, along with the bone flap.

Even though most concerns of scalp deformities (temporal depression, skin indentation) linked to craniotomies have decreased since the introduction of miniplating systems and burr hole covers, the pterional approach remains prone to it, especially surrounding the keyhole, due to the complex curvature of the circumferential bone. Moscovici et al. suggested that a craniotomy without the classical MacCarty keyhole leads to superior aesthetic outcomes with minimal bone loss [15]. Their approach involves delicate sphenoid ridge drilling in a plane parallel to the skull base, thus no longer requiring expansion of the bony window basally.

\section{References}

1. Yasargil MG, et al. Microsurgical pterional approach to aneurysms of the basilar bifurcation. Surg. Neurology. 1976;6:83-91.

2. Weil AG, Robert T, Alsaiari S, Obaid S, Bojanowski MW. Using the trans-lamina terminalis route via a pterional approach to resect a retrochiasmatic craniopharyngioma involving the third ventricle. Neurosurg Focus. 2016;40 Video Suppl 1, 1. 
3. Tayebi Meybodi A, Benet A, Lawton MT. Microsurgical clipping of bilateral superior hypophyseal artery aneurysms through unilateral pterional craniotomy: 3-dimensional operative video. Neurosurgery. 2016;12(2):193.

4. Chaddad-Neto F, et al. The pterional craniotomy: tips and tricks. Arq Neuropsiquiatr. 2012;70:727-32.

5. Miyazawa T. Less invasive reconstruction of the temporalis muscle for pterional craniotomy: modified procedures. Surg Neurol. 1998;50:347-51; discussion 351.

6. Yaşargil MG, Reichman MV, Kubik S. Preservation of the frontotemporal branch of the facial nerve using the interfascial temporalis flap for pterional craniotomy. Technical article. J Neurosurg. 1987;67: 463-6.

7. Altay T, Couldwell WT. The frontotemporal (pterional) approach: an historical perspective. Neurosurgery. 2012;71:481-91; discussion 491-492

8. Andaluz N, Van Loveren HR, Keller JT, Zuccarello M. Anatomic and clinical study of the orbitopterional approach to anterior communicating artery aneurysms. Neurosurgery. 2003;52:1140-8; discussion 1148-1149.
9. Olivas A, Garcia L. 3D neurosurgical approaches. 2011:1.

10. Choi SY, et al. Necessity of surgical site closed suction drain for pterional craniotomy. J Cerebrovasc Endovasc Neurosurg. 2015;17:194-202.

11. Campero A, et al. Pterional transsylvian-transinsular approach in three cavernomas of the left anterior mesiotemporal region. Clin Neurol Neurosurg. 2015;130:14-9.

12. de Andrade Júnior FC, de Andrade FC, de Araujo Filho CM, Carcagnolo Filho J. Dysfunction of the temporalis muscle after pterional craniotomy for intracranial aneurysms. Comparative, prospective and randomized study of one flap versus two flaps dieresis. Arq Neuropsiquiatr. 1998;56:200-5.

13. Spetzler RF, Lee KS. Reconstruction of the temporalis muscle for the pterional craniotomy. Technical note. J Neurosurg. 1990;73:636-7.

14. Kim E, Delashaw JB. Osteoplastic pterional craniotomy revisited. Neurosurgery. 2011;68:125-9; discussion 129.

15. Moscovici S, Mizrahi CJ, Margolin E, Spektor S. Modified pterional craniotomy without 'MacCarty keyhole'. J Clin Neurosci. 2016;24:135-7.

Open Access This chapter is licensed under the terms of the Creative Commons Attribution 4.0 International License (http://creativecommons.org/licenses/by/4.0/), which permits use, sharing, adaptation, distribution and reproduction in any medium or format, as long as you give appropriate credit to the original author(s) and the source, provide a link to the Creative Commons license and indicate if changes were made.

The images or other third party material in this chapter are included in the chapter's Creative Commons license, unless indicated otherwise in a credit line to the material. If material is not included in the chapter's Creative Commons license and your intended use is not permitted by statutory regulation or exceeds the permitted use, you will need to obtain permission directly from the copyright holder. 